\title{
Estimación de la temperatura de punto caliente en transformadores de potencia inmersos en aceite mineral utilizando regresión con vectores de soporte
}

\author{
Andrés F. Cerón(1), Roque A. Lozano(1), Guillermo Aponte(1) y Andrés A. Romero(2) \\ (1) Grupo de Investigación en Alta Tensión, GRALTA., Universidad del Valle, Ciudad Universitaria Meléndez, \\ Calle 13 N 100-00, Cali-Colombia. (correo-e: andres.ceron@correounivalle.edu.co; \\ roque.lozano@correounivalle.edu.co; guillermo.aponte@correounivalle.edu.co). \\ (2) Instituto de Energía Eléctrica, IEE-UNSJ-CONICET., CONICET, Universidad Nacional de San Juan, \\ Av. Lib. San Martín 1109 (Oeste), San Juan-Argentina. (correo-e: aromero@iee-unsjconicet.org).
}

Recibido Nov. 6, 2019; Aceptado Ene. 6, 2020; Versión final Mar. 4, 2020, Publicado Ago. 2020

\begin{abstract}
Resumen
En este artículo se presenta un desarrollo metodológico novedoso para la estimación de la temperatura de punto caliente en transformadores de potencia inmersos en aceite mineral, utilizando regresión con vectores de soporte (RVS). El algoritmo RVS se fundamenta en la teoría de aprendizaje estadístico y hace parte de las herramientas de aprendizaje automático, fue utilizado mediante la implementación de seis etapas, donde se obtiene un modelo de RVS capaz de estimar la variable bajo estudio. El método fue aplicado a un transformador real de 30 MVA con refrigeración ONAN/ONAF al 70/100 \% de carga, donde se utilizó una base de datos para un periodo de 10 años. La validación del modelo de RVS desarrollado se realizó comparando con los resultados obtenidos del modelo Dejan Susa, usando métricas estadísticas de desempeño. En conclusión, los resultados obtenidos indican que la RVS implementada permite estimar con alta exactitud la temperatura de punto caliente.
\end{abstract}

Palabras clave: regresión con vectores de soporte; temperatura de punto caliente; modelo termoeléctrico; transformador de potencia

\section{Hot spot temperature estimation in mineral oil immersed power transformers using support vectors regression}

\begin{abstract}
This article presents an innovative methodological development for the hot spot temperature estimation in mineral oil immersed power transformers by using the support vector regression (SVR). The SVR algorithm is based on the statistical learning theory and is part of the machine learning tools. It was used through a six stage implementation where an SVR model capable of estimating the variable under study is obtained. The method was applied to a real 30 MVA transformer with ONAN/ONAF cooling at 70/100 \% of load using a database for a 10 year period. The developed SVR model was validated by comparison to the results obtained with the Dejan Susa model using statistical performance metrics. In conclusion, the results obtained indicate that the implemented SVR model allows estimating the hot spot temperature with high accuracy.
\end{abstract}




\section{INTRODUCCIÓN}

Los Sistemas de Suministro de Energía Eléctrica (SSEE) son cada vez más importantes en las regiones, tanto en el ámbito social como en el económico, esto ha convertido a la energía eléctrica en un eje estratégico de desarrollo mundial (Agudelo et al., 2014). El transformador de potencia es uno de los elementos más importantes dentro del SSEE. Este equipo es de un costo elevado, alcanzando hasta el $60 \%$ del total de la inversión de una subestación eléctrica, además es fundamental para la operación de las redes de transmisión y distribución de electricidad (Naderian et al., 2008). La falla o mal funcionamiento genera impactos negativos en la red que opera, tales como: pérdida del suministro de energía, sobrecarga de otros elementos, alteraciones en la estabilidad del SSEE, costos por reparación o sustitución de la unidad, entre otros (Medina et al., 2015). En la actualidad, un gran número de transformadores de los SSEE tienen bastantes años de uso y algunos se encuentran cerca de su final de vida útil, lo que conlleva a que las compañías dueñas o administradoras de estos activos, estén interesadas en conocer el tiempo de vida remanente y la condición de los mismos (Cerón et al., 2015a, 2015b).

La temperatura del punto más caliente o TPMC es de importancia en la estimación de vida remanente en transformadores de potencia, debido a que es uno de los factores que afectan directamente la degradación de sus aislamientos y por ende la vida útil del mismo (Cerón et al., 2015a; Martínez et al., 2019). En la literatura, existen diferentes metodologías para obtener la temperatura de punto caliente, las más reconocidas son el modelo termoeléctrico de Dejan Susa (Susa et al., 2005; Susa y Lehtonen, 2006a; Susa y Lehtonen, 2006b), el modelo de ecuaciones exponenciales y diferenciales de la norma IEC 60076-7 (2018) y el modelo de ecuaciones exponenciales de la norma IEEE Std. C57.91 (2011).

La IEC 60076-7 (2018) presenta el modelo térmico dinámico de Susa et al. (2005) como un desarrollo adicional, el cual considera la influencia de la temperatura en la viscosidad del aceite y ha sido verificado físicamente. Sin embargo y aunque el modelo de Susa permite estimar el histórico de TPMC considerando la dinámica del sistema de aislamiento en función del tiempo, a partir del historial de funcionamiento (carga atendida, temperatura ambiente), datos de la placa de características y algunos parámetros del protocolo de la prueba de calentamiento (Romero et al., 2016); requiere la solución de dos ecuaciones diferenciales para un instante de tiempo ti, obteniendo una TPMC $C_{\text {ti. }}$ (Susa et al., 2005; Susa y Lehtonen, 2006a; Susa y Lehtonen, 2006b). Este procedimiento debe ser repetido para todo el período del histórico de funcionamiento del transformador, implicando el uso de un recurso computacional robusto y tiempos de procesamiento bastante importantes. Por ejemplo, en Medina et al. (2015), se presenta una comparación entre los diferentes métodos para calcular la temperatura en el punto más caliente, donde se evidencia que los tiempos de cálculo requeridos por el modelo de Susa, son entre 3500 y 6000 veces superiores a los tiempos requeridos por el modelo de ecuaciones exponenciales, lo cual representa un problema mayor, si se considera que el cálculo del envejecimiento deba resolverse en tiempo real (Mharakurwa et al., 2019).

Por lo tanto, en este artículo se propone utilizar la herramienta Regresión con Vectores de Soporte o RVS, para estimar la temperatura de punto caliente, reduciendo los tiempos de obtención de la estimación, con respecto al modelo de Susa, asegurando el rendimiento de la RVS mediante métricas estadísticas.

\section{TEMPERATURA DE PUNTO CALIENTE}

En los transformadores de potencia en servicio, interactúan esencialmente fenómenos térmicos y eléctricos, siendo el fenómeno térmico resultado de la carga dinámica y las condiciones ambientales variables (Romero et al., 2016; Mharakurwa et al., 2019). El estrés térmico es el mayor causante del deterioro del sistema de aislamiento del transformador, en especial del aislamiento sólido. Sin embargo, el deterioro del aislamiento sólido no sucede de manera uniforme en los devanados de los transformadores de potencia, sino que se concentra en zonas puntuales, específicamente en el denominado "Punto Caliente" (Hot Spot - HS del inglés) (Medina et al., 2015). En tal punto es el lugar donde se produce el mayor envejecimiento del aislamiento sólido por degradación térmica (Medina et al., 2017). La TPMC es de gran utilidad para estimar el envejecimiento del aislamiento solido debido al efecto térmico, dada la criticidad que tiene sobre el mismo.

\section{Modelo termoeléctrico de Dejan Susa}

El modelo termoeléctrico para estimar la TPMC fue desarrollado por Susa en su tesis doctoral (Susa, 2005) y publicado en tres artículos (Susa et al., 2005; Susa y Lehtonen, 2006a; Susa y Lehtonen, 2006b). Este modelo se sustenta en la analogía de dos teorías convencionales, la de transferencia de calor por medio de fluidos y la de circuitos eléctricos respectivamente. Además, el modelo establece que es posible relacionar matemáticamente la interacción de los fenómenos térmicos y eléctricos. En la Fig. 1 se presenta 
simplificadamente el circuito eléctrico y su equivalente térmico, del modelo. En la Tabla 1 se relacionan los símbolos utilizados en el diagrama de la Fig. 1.
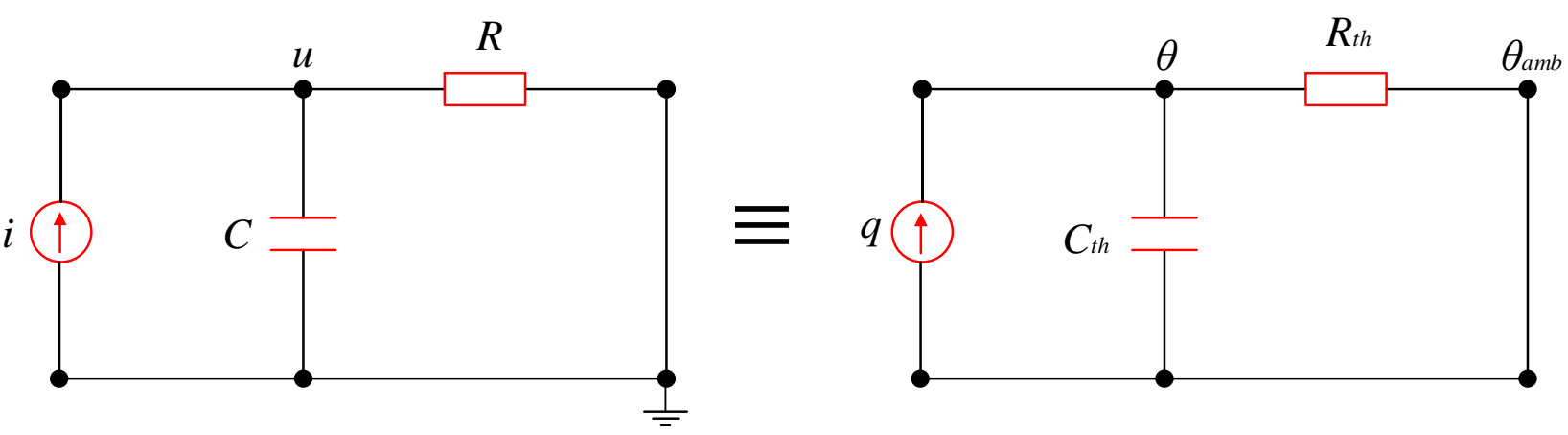

Fig. 1: Diagrama básico del equivalente termoeléctrico. (Adaptada de Susa, 2005).

Tabla 1: Variables equivalentes de la analogía termoeléctrica.

\begin{tabular}{|c|c|c|c|c|c|}
\hline \multicolumn{2}{|c|}{ Equivalente Eléctrico } & \multicolumn{3}{c|}{ Equivalente Térmico } \\
\hline Parámetros & Símbolo & Unidad & Parámetros & Símbolo & Unidad \\
\hline Corriente & $i$ & $\mathrm{~A}$ & Calor generado & $q$ & $\mathrm{~W}$ \\
\hline Voltaje & $u$ & $\mathrm{~V}$ & Temperatura & $\theta$ & ${ }^{\circ} \mathrm{C}$ \\
\hline Resistencia & $R$ & $\Omega$ & Resistencia & $R_{\text {th }}$ & ${ }^{\circ} \mathrm{C} / \mathrm{W}$ \\
\hline Capacitancia & $\mathrm{C}$ & $\mathrm{F}$ & Capacitancia & $C_{\text {th }}$ & $\mathrm{J} /{ }^{\circ} \mathrm{C}$ \\
\hline Nodo de referencia & Gnd & $\mathrm{V}$ & Temperatura ambiente & $\theta_{\text {amb }}$ & ${ }^{\circ} \mathrm{C}$ \\
\hline
\end{tabular}

De la Fig. 1 en el equivalente eléctrico, $i$ es la corriente, $C$ es la capacitancia, $R$ es la resistencia y $u$ es el voltaje; del equivalente térmico, $q$ es el calor generado, $C_{\text {th }}$ es la capacitancia térmica, $\theta$ es la temperatura, $R_{t h}$ es la resistencia térmica y $\theta_{a m b}$ es la temperatura ambiente (Susa, 2005). $R_{t h}$ y $C_{t h}$ representan la capacidad del material de resistir y almacenar calor, respectivamente (Susa, 2005). El fenómeno eléctrico se define matemáticamente por la teoría de circuitos $\mathrm{RC}$ basada en la ley de Ohm y las reglas de Kirchhoff, y el térmico, por la ecuación de balance energético (Susa, 2005).

Este modelo estima la TPMC a partir de la carga atendida, la temperatura ambiente, datos de placa y algunos parámetros de la prueba de calentamiento. Esto se logra mediante la solución de las ecuaciones diferenciales (1) y (2) (Susa y Lehtonen, 2006a; Susa y Lehtonen, 2006b; Medina et al., 2017). La primera representa la temperatura del aceite en la parte superior del transformador $\theta_{\text {Tо }}$ y la segunda, la temperatura del punto más caliente $\theta_{\text {HS }}$.

$$
\begin{aligned}
& {\left[\left(\frac{1+R \cdot K^{2}}{1+R}\right) \cdot \mu_{\mathrm{PU}}{ }^{\mathrm{n}} \cdot \Delta \theta_{\mathrm{TO}, \mathrm{R}}\right]=\left[\mu_{\mathrm{PU}}{ }^{\mathrm{n}} \cdot \tau_{\mathrm{TO}, \mathrm{R}} \cdot \frac{\mathrm{d} \theta_{\mathrm{oil}}}{\mathrm{dt}}+\frac{\left(\theta_{\mathrm{TO}}-\theta_{\mathrm{amb}}\right)^{\mathrm{n}+1}}{\Delta \theta_{\mathrm{TO}, \mathrm{R}}{ }^{\mathrm{n}}}\right]} \\
& {\left[K^{2} \cdot \mu_{\mathrm{PU}}{ }^{\mathrm{m}} \cdot \Delta \theta_{\mathrm{HS}, \mathrm{R}}\right]=\left[\mu_{\mathrm{PU}}{ }^{\mathrm{m}} \cdot \tau_{\mathrm{W}, \mathrm{R}} \cdot \frac{\mathrm{d} \theta_{\mathrm{HS}}}{\mathrm{dt}}+\frac{\left(\theta_{\mathrm{HS}}-\theta_{\mathrm{TO}}\right)^{\mathrm{m}+1}}{\Delta \theta_{\mathrm{HS}, \mathrm{R}}^{\mathrm{m}}}\right]}
\end{aligned}
$$

Donde: $K$ es el factor de carga descrito por el cociente entre la carga y la carga nominal, $R$ es la relación entre el valor pérdidas bajo carga y de vacío, $\theta_{\mathrm{amb}}$ es la temperatura ambiente, $\Delta \theta_{\mathrm{TO}, \mathrm{R}}$ es el incremento de la temperatura del aceite de la parte superior sobre $\theta_{\mathrm{amb}}, \Delta \theta_{\mathrm{HS}, \mathrm{R}}$ es el incremento de la temperatura de punto más caliente sobre $\theta_{\mathrm{amb}}$, Tто-R es la constante de tiempo termodinámica del aceite superior, Tw- $\mathrm{R}$ es la constante de tiempo termodinámica del devanado, $\mu_{P U}$ es la viscosidad del aceite en por unidad, $n$ es una constante empírica que depende del tipo de circulación del aceite y $m$ es una constante empírica que modela el comportamiento térmico no lineal de los devanados de la unidad. Para el modo de enfriamiento 
ONAN, el valor de las constantes $n$ y $m$ es 0.25 . Para los modos de enfriamiento ONAF y OFAF, en transformadores de potencia con enfriamiento externo estos valores son $n=0.5$ y m $=0.1$ (Susa, 2005).

\section{ESTIMACIÓN DE LA TEMPERATURA DE PUNTO CALIENTE}

En esta sección se propone un método para estimar la TPMC en transformadores de potencia que han estado en funcionamiento, utilizando su información histórica. Las variables de interés son: parámetros característicos del transformador bajo estudio, y los registros históricos de temperatura ambiente (en ${ }^{\circ} \mathrm{C}$ ) y potencia activa atendida (en MW). Antes de exponer el desarrollo metodológico, se realiza una descripción general de la RVS y de las métricas estadísticas para medir el desempeño.

\section{Regresión con Vectores de Soporte - RVS}

La RVS es un método computacional basado en la teoría de aprendizaje estadístico, que permite realizar aportes a la fiabilidad, la seguridad y la eficiencia de la red eléctrica (Estupiñan et al, 2016; Gómez et al., 2018). Puede resolver el problema de los extremos excesivos y locales, causados por el algoritmo de evolución (Mosquera et al., 2018). Sin embargo, no es fácil seleccionar los parámetros que influyen en la precisión de la estimación (Qian et al., 2017; Cui et al., 2015). Básicamente la RVS se estructura a partir de un conjunto de muestras de entrenamiento $\left\{\left(X_{i}, Y_{i}\right), i=1,2,3, \ldots,\right\}$, donde $X_{i}$ es el vector de características de entrenamiento, $Y_{i}$ es el valor de salida y $/$ es el número total de datos. Por simplicidad, se representa matemáticamente por una función de regresión lineal, ver la ecuación (3) (Qian et al., 2017; Cui et al., 2015).

$$
y^{\prime}=\mathrm{w}^{T} \cdot \varphi(x)+b
$$

Donde $\mathrm{w}^{\top}$ es el vector de pesos transpuesto, $b$ es el término de sesgo, $\varphi$ es la representación del núcleo (kernel del inglés) y y' es el valor predicho. La RVS intrínsecamente es un problema de optimización con sus respectivas restricciones, como se observa en la ecuación (4) (Qian et al., 2017; Cui et al., 2015).

$$
\begin{aligned}
\operatorname{mín} \frac{1}{2}\|\mathrm{w}\|^{2}+\mathrm{C} \cdot \sum_{\mathrm{i}=1}^{\mathrm{n}}\left(\xi_{\mathrm{i}}+\xi_{\mathrm{i}}^{*}\right) \text { sujeto } \mathrm{a}\left[w \cdot \Phi\left(x_{i}\right)+b\right] \leq \varepsilon+\xi_{i} \\
{\left.\left[x_{i}\right)+b\right]-\mathrm{y}_{\mathrm{i}} \leq \varepsilon+\xi_{\mathrm{i}}^{*} } \\
\xi_{i} \geq 0, \xi_{\mathrm{i}}^{*} \geq 0 ; i=1, \ldots, n
\end{aligned}
$$

En la ecuación (4), C es mayor que 0 y representa un factor de penalización para la regularización, épsilon $(\varepsilon)$ es mayor que cero y representa la tolerancia de desviación, $\xi_{i}$ y $\xi_{i}^{*}$ son variables de control para la aproximación del regresor RVS (Qian et al., 2017; Cui et al., 2015). Al introducirle multiplicadores de Lagrange $\alpha_{i}$ y $\alpha_{i}{ }^{*}$ al problema de optimización, la RVS se transforma en un problema de optimización cuadrático dual, además, adicionando la noción de núcleo $\mathrm{K}\left(\mathrm{x}_{\mathrm{i}}, \mathrm{y}_{\mathrm{i}}\right)=\varphi\left(\mathrm{x}_{\mathrm{i}}\right) \varphi\left(\mathrm{x}_{\mathrm{j}}\right)$, se obtiene la función que se muestra en la ecuación (5) (Qian et al., 2017; Cui et al., 2015).

$y^{\prime}=\sum_{i=1}^{n}\left(\alpha_{i}-\alpha_{i}^{*}\right) K\left(x_{i}, y_{i}\right)+b$

La RVS se puede usar para regresión no lineal utilizando varias nociones de núcleo, entre las cuales las funciones más utilizadas son: la lineal, la de base radial gaussiana (BRG), la polinomial y los núcleos de perceptrón multicapa (Qian et al., 2017; Cui et al., 2015). En este estudio, se utilizó la función núcleo lineal dada su simplicidad y aplicabilidad en modelos de regresión, además, se ha demostrado que en ocasiones puede superar a modelos no lineales más complejos (Hastie et al., 2009).

\section{Métricas estadisticas de desempeño}

El análisis de errores, utilizando métricas de desempeño, es uno de los pasos importantes para probar modelos de estimación. A través del cálculo de las métricas, las ventajas y desventajas del modelo RVS pueden evaluarse estadísticamente, permitiendo cuantificar su eficiencia en la estimación del TPMC. En este artículo, tres métricas estadísticas fueron utilizadas para evaluar el desempeño del modelo RVS desarrollado (Deng et al., 2019; Cortés et al., 2010), las cuales son: 1) Raíz del Error Cuadrático Medio RECM, ver ecuación (6). 2) Error Absoluto Porcentual Promedio - EAPP, ver ecuación (7). 3) Índice de Correlación de Concordancia - ICC, ver ecuación (8). 


$$
\begin{aligned}
& R E C M=\sqrt{\frac{1}{l} \cdot \sum_{i=1}^{l}\left(Y_{i}{ }^{\prime}-Y_{i}\right)^{2}} \quad\left[{ }^{\circ} C\right] \\
& E A P P=\frac{1}{l} \cdot \sum_{i=1}^{l}\left(\frac{\left|Y_{i}{ }^{\prime}-Y_{i}\right|}{Y_{i}} \cdot 100\right) \quad[\%]
\end{aligned}
$$

En las ecuaciones (6) y (7), l es el tamaño del vector $Y_{i}$ o $Y_{i}^{\prime} ; Y_{i}^{\prime}$ es el valor de la variable estimada en el instante $i$; e $Y_{i}$ es el valor verdadero de la variable en el instante $i$.

$$
\mathrm{ICC}=\frac{\mathrm{S}_{\mathrm{Y} 1}{ }^{2}+\mathrm{S}_{\mathrm{Y} 2}{ }^{2}-\mathrm{S}_{\mathrm{Y} 1}-\mathrm{Y}^{2}}{\mathrm{~S}_{\mathrm{Y} 1}{ }^{2}+\mathrm{S}_{\mathrm{Y} 2}{ }^{2}+\left(\overline{\mathrm{Y}}_{1}-\overline{\mathrm{Y}}_{2}\right)^{2}}
$$

En la ecuación (8), $S_{Y 1}{ }^{2}$ : es la varianza del modelo de regresión; $S_{Y 2}{ }^{2}$ : es la varianza de la variable verdadera; $\mathrm{S}_{Y 1-\mathrm{Y}_{2}}$ : es la varianza de la diferencia promedio entre el modelo de regresión y la variable verdadera; e $\bar{Y}_{1}-\bar{Y}_{2}$ : es la diferencia promedio del modelo de regresión y la variable verdadera.

\section{Estimación de la TPMC con RVS}

El procedimiento para estimar el TPMC en transformadores de potencia utilizando RVS es el siguiente:

(1) Adecuación de la base de datos: se depura y adecua la información histórica de funcionamiento (potencia atendida, temperatura ambiente) y datos requeridos para estimar la TPMC con el modelo de Susa.

(2) TPMC con Modelo de Susa: se calcula el histórico de TPMC de un transformador. En caso de tener la medición directa del TPMC, este paso puede ser omitido.

(3) Normalización de la base datos: se eliminan los efectos de las diferentes magnitudes y dimensiones que presentan las variables bajo estudio (potencia, temperatura ambiente y la TPMC-Susa). Las variables se normalizan dentro de un rango de 0 a 1 (Deng et al., 2019), utilizando la ecuación (9).

$$
X_{i}^{\prime}=\frac{x_{i}-x_{\min }}{x_{\max }-x_{\min }}
$$

Donde $x_{i}$ es el valor i-esimo de la variable $X, X_{\min }$ y $X_{\max }$ son el valor mínimo y máximo, y $X_{i}^{\prime}$ es el valor normalizado.

(4) Entrenamiento y prueba de RVS: se determina un subconjunto de la base de datos para entrenar el modelo de RVS (entrenamiento supervisado), configurado con un núcleo lineal, empleando parámetros adecuados del factor de penalización $C$ y la tolerancia $\varepsilon$. Una vez entrenado el modelo, se implementa para obtener el histórico de TPMC $_{\mathrm{t} j}$ para un subconjunto de prueba $\mathrm{X}_{\mathrm{j}}$. Los datos estimados se desnormalizan para obtener el histórico en unidades reales.

(5) Métricas estadísticas de desempeño: se calcula el RECM, EAPP e ICC para el conjunto de datos obtenidos en la prueba del modelo RVS desarrollado.

6) Análisis del modelo: mediante las métricas y el análisis visual, se evalúa el desempeño general del modelo de RVS comparando contra el perfil de TPMC obtenido por Susa y se aprueba o no, para su utilización.

\section{CASO DE ESTUDIO}

En esta sección se presenta la aplicación del modelo de RVS propuesto, para estimar la temperatura de punto caliente a un transformador de potencia de 30 MVA, inmerso en aceite mineral y que ha estado en operación desde el año 1969. Los resultados obtenidos son comparados con el modelo termoeléctrico de Susa (2005), finalmente se evalúa el rendimiento del modelo RVS desarrollado, siguiendo el procedimiento metodológico descrito en la sección anterior. 


\section{Característica del transformador bajo estudio}

Los datos requeridos para la estimación de la TPMC del transformador bajo estudio, son presentados en la Tabla 2, estos datos corresponden a un transformador trifásico, tridevanado, con una potencia de 30/30/20 MVA, un nivel de tensión de $132 \pm 5-20 \% / 34.5 \pm 2.5-5 \% / 13.8 \mathrm{kV}$, conectado en $\mathrm{Yn} / \mathrm{Yn} 0 / \mathrm{D} 11$ y refrigeración ONAN/ONAF para el $70 / 100 \%$ de la carga.

Tabla 2: Parámetros requeridos para el modelamiento de la TPMC del transformador bajo estudio.

\begin{tabular}{|l|l|l|l|}
\hline Sn $[\mathrm{MVA}]$ & $30 / 30 / 20$ & $\mathrm{~m}_{\mathrm{o}}[\mathrm{kg}]$ & 20277 \\
\hline $\mathrm{Vn}[\mathrm{kV}]$ & $132 \pm 5-20 \% / 34.5 \pm 2.5-5 \% / 13.8$ & Perdidas vacío $[\mathrm{W}]$ & 20000 \\
\hline Conexión & Yn/Yn0/D11 & Perdidas carga $[\mathrm{W}]$ & 165000 \\
\hline Refrigeración & ONAN/ONAF $(70 / 100 \%$ de la carga) & $R$ [adimensional] & 8.25 \\
\hline$\tau_{\mathrm{W}-\mathrm{R}}[\mathrm{min}]$ & 7 & $\tau_{\mathrm{TO}-\mathrm{R}}[\mathrm{min}]$ & 157.83 \\
\hline$\Delta \theta_{\mathrm{TO}, \mathrm{R}}\left[{ }^{\circ} \mathrm{C}\right]$ & 50 & $\theta_{\mathrm{TO}, \mathrm{R}}\left[{ }^{\circ} \mathrm{C}\right]$ & 75 \\
\hline$\Delta \theta_{\mathrm{HS}, \mathrm{R}}\left[{ }^{\circ} \mathrm{C}\right]$ & 20 & $\theta_{\mathrm{HS}, \mathrm{R}}\left[{ }^{\circ} \mathrm{C}\right]$ & 95 \\
\hline $\mathrm{C}_{\mathrm{o}}\left[\mathrm{Wh} /{ }^{\circ} \mathrm{C}\right]$ & 9733 & $\theta_{\mathrm{amb}, \mathrm{R}}\left[{ }^{\circ} \mathrm{C}\right]$ & 40 \\
\cline { 1 - 2 }$\delta$ Densidad del aceite $\left[\mathrm{kg} / \mathrm{m}^{3}\right]$ & 0.851 & \multicolumn{2}{|c}{} \\
\cline { 1 - 3 } & &
\end{tabular}

Las temperaturas y sus gradientes, se pueden obtener mediante el uso de termocuplas o sensores de temperatura de fibra óptica (IEC 60076-7, 2018; IEEE Std. C57.91, 2011; Susa, 2005). Aunque la mayoría de los parámetros necesarios para poder ejecutar el modelo de temperatura de punto caliente se pueden determinar en función de las características de la placa de identificación del transformador bajo estudio, el modelo de temperatura de punto caliente de Susa requiere datos de la prueba de ejecución de calor que pueden no estar disponibles, En ese caso, se puede trabajar con valores típicos, con base en la Tabla 3 , según la IEC 60076-7 (2018). Donde un transformador pequeño se refiere a aquellas unidades de potencia sin radiadores, refrigeradores o tubos conectados, independientemente de la capacidad nominal; transformador de mediana potencia son aquellos con una clasificación máxima de 100 MVA trifásica o 33.3 MVA monofásica; y finalmente un transformador de alta potencia se refiere a las unidades con una capacidad nominal máxima de más de 100 MVA trifásica o de más de 33.3 MVA monofásica.

Tabla 3: Valores típicos de parámetros para la estimación de la TPMC para ONAN y ONAF (IEC60076-7, 2018).

\begin{tabular}{|c|c|c|c|}
\hline \multirow{2}{*}{ Característica } & Transformadores pequeños & Transformadores de mediana y alta potencia \\
\cline { 2 - 4 } & ONAN & ONAN & ONAF \\
\hline$\tau_{\text {TO-R }}[\mathrm{min}]$ & 180 & 210 & 150 \\
\hline$\tau_{\mathrm{W}-\mathrm{R}[\mathrm{min}]}$ & 4 & 10 & 7 \\
\hline
\end{tabular}

Por lo anterior, el valor de la constante de tiempo termodinámica del devanado se tomó como un valor típico obtenido de la Tabla 3, obteniendo que $\tau$ w-R=7 [min].

El valor de la constante termodinámica del aceite superior se obtuvo por medio de la ecuación (10) (Susa y Lehtonen, 2006a).

$\tau_{T O, R}=C_{O} \cdot \frac{\Delta \theta_{T O, R}}{P_{T, R}} \cdot 60$

Donde $\mathrm{C}_{\circ}$ es la capacidad térmica del aceite en $\left[\mathrm{Wh} /{ }^{\circ} \mathrm{C}\right]$, y se define en la ecuación (11); $\mathrm{P}_{\mathrm{T}, \mathrm{R}}$ son las pérdidas totales en $[\mathrm{W}]$.

$$
C_{O}=0.48 \cdot m_{o}
$$

Donde $\mathrm{m}_{\mathrm{o}}$ es el peso del aceite en $[\mathrm{kg}]$.

Para el cálculo de $\theta_{\mathrm{HS}}$, se dispone de los históricos de temperatura ambiente y de potencia atendida, ambos para un periodo comprendido del 20-Ene-2008 al 01-Ene-2019. Para cada día (del periodo) se tienen 48 registros de las dos variables, con un paso de muestra de $30 \mathrm{~min}$; resultando un total de 191951 muestras para el periodo indicado. 
Para este periodo de tiempo, es necesario aclarar que el transformador estuvo alternando su modo de refrigeración entre ONAN y ONAF, con base en su nivel de carga. Por esta razón y para poder estimar correctamente la TPMC, se debe modelar la señal que acciona los ventiladores de la unidad evaluada, es decir, que inicia o suspende el modo ONAF, la cual está dada por un monitor de temperatura comercial, que sigue la formulación de control de la ecuación (12) (Romero et al., 2016).

$$
\theta_{H S, c}=\theta_{T O}+H \cdot \Delta \theta_{H S, R} \cdot K^{x}
$$

Donde, $\mathrm{H}$ y $x$ son valores que dependen de las características del transformador, de hecho, en este estudio de caso, $\mathrm{H}=1.3$ y $x=1.6$. Cabe señalar que la señal de control de la TPMC por medio $\theta_{\mathrm{HS}, \mathrm{c}}$ (ecuación (12)) es una aproximación, cuyo único propósito es accionar los ventiladores. De hecho, los ventiladores se encienden cuando $\theta_{\mathrm{HS}, \mathrm{C}}$ es superior a $85^{\circ} \mathrm{C}$, y se apagan cuando $\theta_{\mathrm{HS}, \mathrm{c}}$ se vuelve inferior a $75{ }^{\circ} \mathrm{C}$ para evitar operaciones repetitivas de encendido y apagado del ventilador. En el algoritmo que se implementó, cuando $\theta$ Tо, calculado a partir de la ecuación (1) y $K$ (que depende de la carga), produce valores de $\theta_{\mathrm{HS}, \mathrm{c}}$ superiores a $85^{\circ} \mathrm{C}$ cuando la temperatura aumenta; valores inferiores a $75^{\circ} \mathrm{C}$ cuando la temperatura está disminuyendo, entonces las constantes empíricas $n$ y $m$ en la ecuación (1) y ecuación (2) cambian y asumen sus valores respectivos de acuerdo con el modo de refrigeración activado, esto para todo el periodo de tiempo de estimación de la TPMC.

\section{RESULTADOS}

Los valores de los parámetros que complementan la función núcleo lineal del modelo RVS desarrollado, C y $\varepsilon$ respectivamente, se presentan en la Tabla 4 . Una vez determinado los parámetros, se entrenaron tres modelos de RVS con el fin de evaluar la sensibilidad del mismo en función del conjunto de datos utilizado en la etapa de entrenamiento. Por lo tanto, se procesó la información de la base de datos de tal forma que cada modelo entrenado utilizara un porcentaje distinto del total de las muestras como conjunto de entrenamiento; cada conjunto fue seleccionado de manera aleatoria y corresponden a un $20 \%, 50 \%$ y $80 \%$ del total de la información. En total se tienen 191951 muestras, donde cada muestra es de tres dimensiones: temperatura ambiente, potencia y TPMC de Susa; las dos primeras son variables de entrada y la tercera es la variable de salida.

Tabla 4: Parámetros utilizados para el desarrollo del modelo RVS.

\begin{tabular}{|l|c|}
\hline \multicolumn{1}{|c|}{ Parámetros } & Valores \\
\hline Factor de penalización C & $1.000 \times 10^{1}$ \\
\hline Épsilon $\varepsilon$ & $1.236 \times 10^{-2}$ \\
\hline
\end{tabular}

Como conjunto de prueba de los modelos, se utilizó el total de la información disponible, es decir, para cada modelo entrenado se estimó el histórico de TPMC para el periodo de análisis (20-Ene-2008 al 01-Ene2019). Los históricos de TPMC obtenidos con los tres modelos, junto al histórico obtenido con el modelo de Susa, son graficados en la Fig. 2, para una muestra de 21 días.

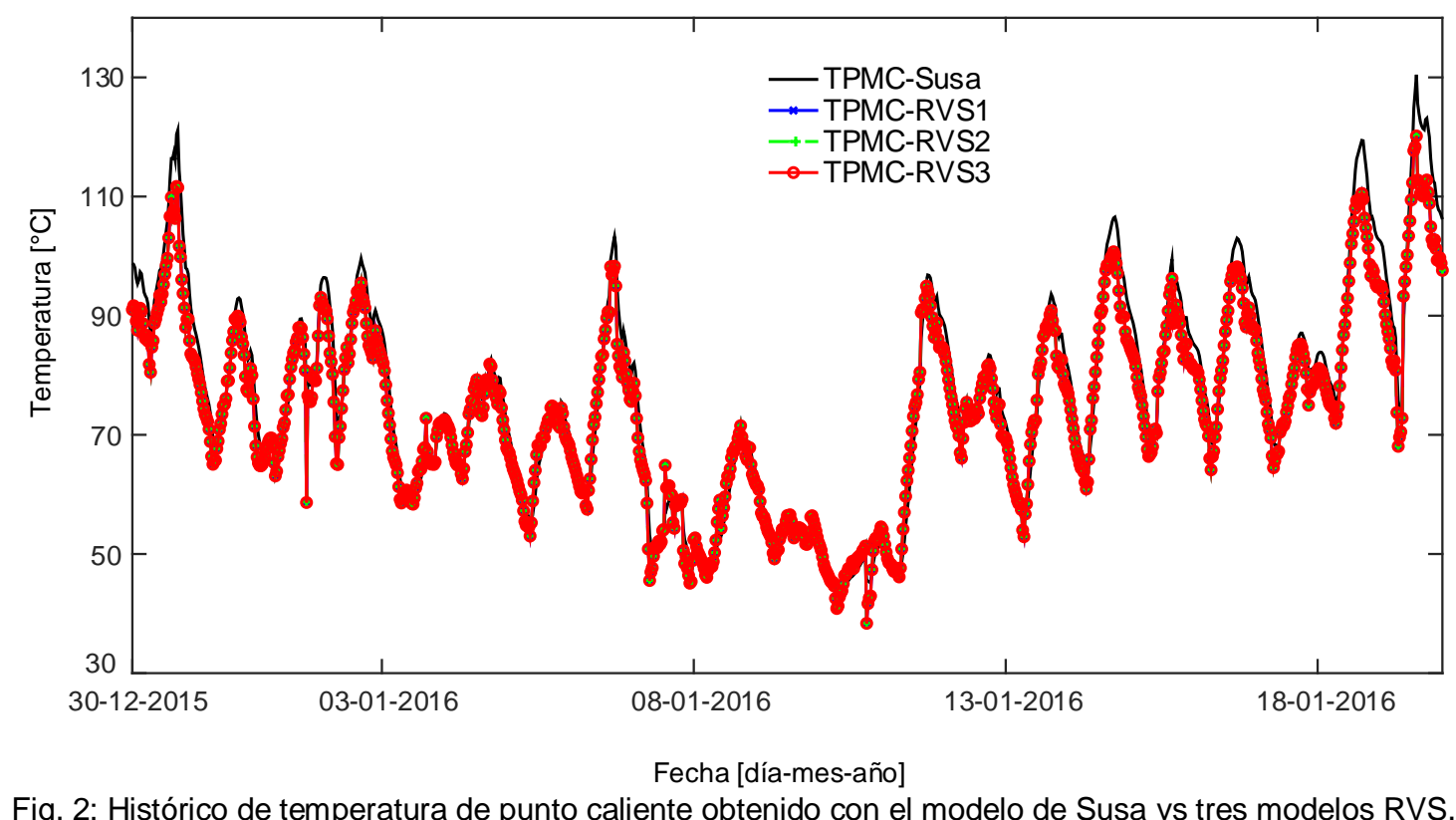


En la Fig. 3, se graficó una ventana de tiempo de treinta y seis (36) horas (dentro del periodo de análisis) con el fin de notar diferencia entre los históricos obtenidos por los modelos, dado que, tomando todo el periodo, las señales quedan visualmente superpuestas. El cálculo de las métricas estadísticas de desempeño e información complementaria para el análisis se presenta en la Tabla 5.

Tabla 5: Matriz de resultados de los modelos.

\begin{tabular}{|c|c|c|c|c|c|c|c|}
\hline Modelo & $\begin{array}{c}\text { Muestras de } \\
\text { entrenamiento } \\
{[\%]}\end{array}$ & $\begin{array}{c}\text { Muestras } \\
\text { de prueba } \\
{[\%]}\end{array}$ & $\begin{array}{c}\text { Tiempo de } \\
\text { entrenamiento } \\
\text { [hh:mm:ss] }\end{array}$ & $\begin{array}{c}\text { Tiempo de } \\
\text { prueba } \\
\text { [hh:mm:ss] }\end{array}$ & RECM [ $\left.{ }^{\circ} \mathrm{C}\right]$ & EAPP [\%] & ICC \\
\hline TPMC-RVS1 & 20 & 100 & $0: 00: 49$ & $0: 00: 07$ & 2.978 & 5.013 & 0.9790 \\
\hline TPMC-RVS2 & 50 & 100 & $0: 04: 08$ & $0: 00: 17$ & 2.980 & 5.008 & 0.9789 \\
\hline TPMC-RVS3 & 80 & 100 & $0: 09: 58$ & $0: 00: 31$ & 2.981 & 5.004 & 0.9789 \\
\hline TPMC-Susa & - & 100 & - & $0: 14: 26$ & - & - & - \\
\hline
\end{tabular}

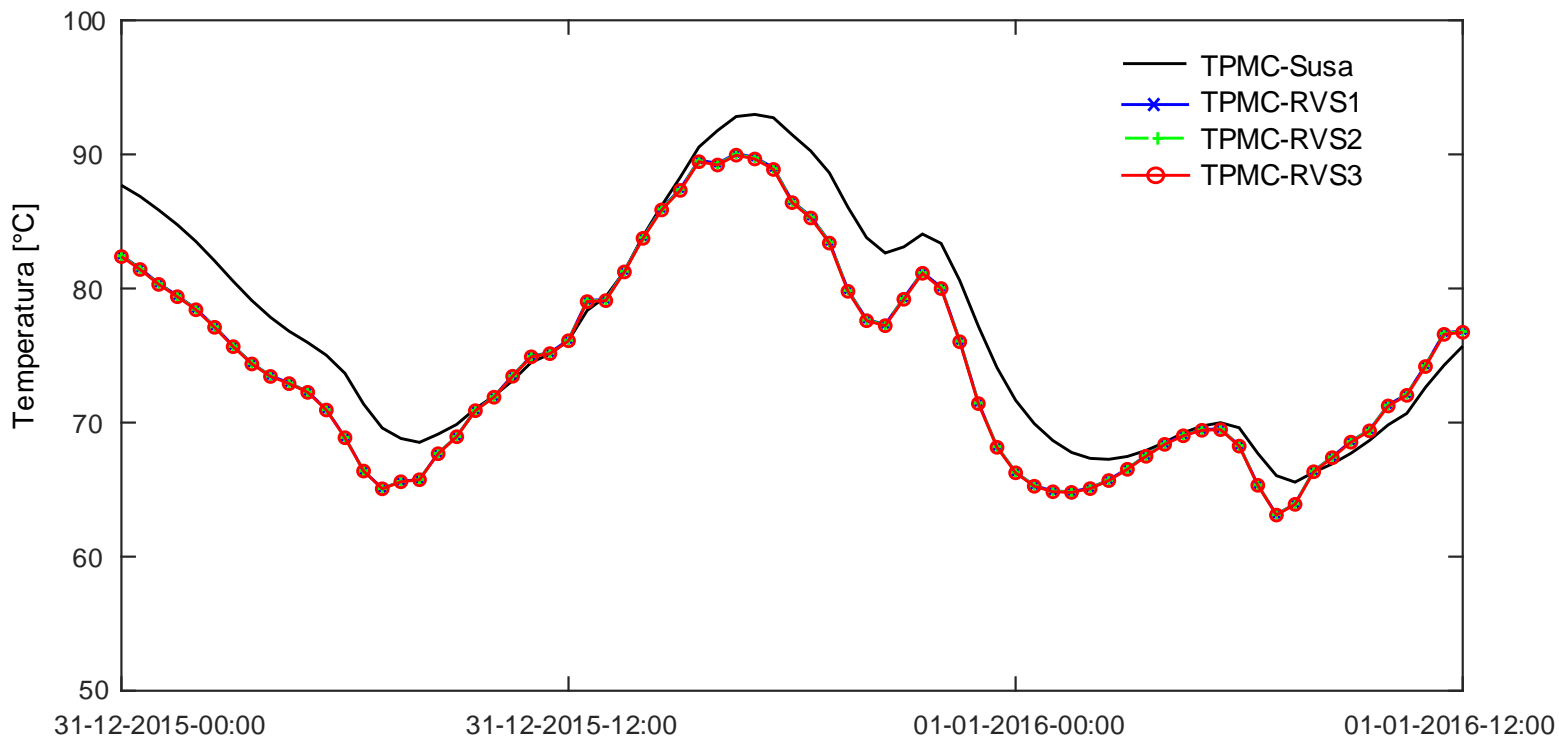

Fecha [día-mes-año-hora]

Fig. 3: Históricos de temperatura de punto caliente obtenidos por los cuatro modelos, ventana de treinta y seis horas.

En general, las métricas de desempeño constatan que los modelos de RVS implementados estiman la TPMC con gran precisión, obteniendo RECM menores a $3{ }^{\circ} \mathrm{C}$ y un EAPP cercano al $5 \%$, lo que demuestra que en promedio las estimaciones difieren de cada TPMC $\mathrm{C}_{\mathrm{ti}}$ obtenido por el modelo de Susa en aproximadamente $3{ }^{\circ} \mathrm{C}$ y que la precisión de las estimaciones son cercanas al 95\%; por su parte el ICC indica que los modelos reproducen sustancialmente el fenómeno bajo estudio (valores entre 0.95 y 0.99 ) y son capaces de reproducir la TPMC del transformador de potencia con gran precisión y exactitud (Cortés et al., 2010).

El modelo "TPMC-RVS1" entrenado con un $20 \%$ de las muestras seleccionadas aleatoriamente, mostró un gran desempeño comparado con los restantes, pues fue el que obtuvo la menor RECM y mayor ICC. Por su parte el modelo "TPMC-RVS3" entrenado con un $80 \%$ de las muestras, fue el que obtuvo menor EAPP, es decir, mejor precisión en las estimaciones.

\section{DISCUSIÓN}

El uso de modelos basados en RVS para la estimación de TPMC permite obtener buenos resultados, tal como se demuestra en Cui et al. (2015) y Deng et al. (2017). En Cui et al. (2015) se propuso un algoritmo hibrido para la estimación del TPMC del transformador integrando RVS con granulación de información (Gl), donde se alcanzó un error relativo máximo de 3.5\%. En Deng et al. (2017) se desarrolló otro método hibrido de RVS con búsqueda exhaustiva (GS) para estimar el TPMC, en el que alcanzan un RECM $1.26{ }^{\circ} \mathrm{C}$ y un EAPP inferior al 1\% para sus estimaciones. En comparación con el desarrollo realizado, donde se alcanzó un error absoluto promedio aproximado al $5 \%$ y un RECM inferior a los $3^{\circ} \mathrm{C}$. 
Los estudios realizados por Cui et al. (2015) y Deng et al. (2017) demuestran que métodos de estimación basados en RVS pueden ser muy eficaces estimando el TPMC en transformadores, superando incluso los métodos tradicionales basados en ecuaciones diferenciales.

Por otro lado, se puede señalar que al igual que los resultados obtenidos en Mosquera (2018), los RVS desarrollados, logran disminuir considerablemente el tiempo de cálculo manteniendo una alta precisión, en este caso, con respecto al modelo de Susa.

La metodología desarrollada, a diferencia de Cui et al. (2015) y Deng et al. (2017), se centra en el aprovechamiento del potencial que trae consigo el algoritmo RVS y la utilización de información del historial operativo de transformadores de potencia que hayan estado en funcionamiento por muchos años dentro de los sistemas de suministro de energía eléctrica.

\section{CONCLUSIONES}

De acuerdo a los resultados obtenidos y la discusión realizada, se demostró que es posible estimar la temperatura de punto caliente de transformadores de potencia, mediante herramientas de aprendizaje automático, específicamente usando regresión con vectores de soporte (RVS) en conjunto con el desarrollo metodológico propuesto. Por otro lado, se logró que la estimación realizada, disminuyera los tiempos de procesamiento con respecto al modelo de Susa. Una vez entrenado el modelo de RVS, es capaz de estimar el histórico de TPMC, para periodos relativamente grandes, en cuestión de segundos.

La metodología propuesta representa una herramienta innovadora para los profesionales expertos de áreas de mantenimiento y gestión de activos de las compañías eléctricas, permitiendo recrear bajo condiciones reales de funcionamiento el histórico de TPMC; el cual es usado para diagnosticar técnicamente el estado mecánico del aislamiento sólido del transformador de potencia.

Finalmente, se expuso mediante las métricas estadísticas de desempeño, que los algoritmos basados en RVS, entrenados de una forma acertada, son eficaces y precisos en la estimación de la temperatura de punto caliente.

\section{AGRADECIMIENTOS}

Los autores desean agradecer a la Universidad del Valle y a Colciencias por su apoyo en este trabajo a través del proyecto "Metodología para la definición de estrategias de sustitución en el largo plazo para transformadores de potencia, considerando criterios técnicos y económicos".

\section{REFERENCIAS}

Agudelo, L., Velilla E. y López J.M., Estimación de la Carga de Transformadores de Potencia Utilizando una Red Neuronal Artificial, http://dx.doi.org/10.4067/S0718-07642014000200003, Información Tecnológica, 25(2), 15-24 (2014).

Cerón, A.F., Echeverry D.F., Aponte G. y Romero A.A., Índice de Salud para Transformadores de Potencia Inmersos en Aceite Mineral con Voltajes entre $69 \mathrm{kV}$ y $230 \mathrm{kV}$ usando Lógica Difusa, http://dx.doi.org/10.4067/S071807642015000200013, Información Tecnológica, 26(2), 107-116 (2015).

Cerón, A.F., Aponte G., Echeverry D.F. y Romero A.A., Panorama de la Gestión de Activos para Transformadores de Potencia, http://dx.doi.org/10.4067/S0718-07642015000300014, Información Tecnológica, 26(3), 99-110 (2015).

Cortés, E., Rubio J. y Gaitán H., Métodos Estadísticos de Evaluación de la Concordancia y la Reproductibilidad de Pruebas Diagnósticas, ISSN 0034-7434, Revista Colombiana de Obstetricia y Ginecología, 61(3), 247-255 (2010).

Cui, Y., Ma H. y Saha T., Transformer Hot Spot Temperature Prediction using a Hybrid Algorithm of Support Vector Regression and Information Granulation, https://doi.org/10.1109/APPEEC.2015.7381066, IEEE PES Asia-Pacific Power and Energy Engineering Conference (APPEEC), 1-5 (2015).

Deng, Y., Ruan J. y otros cinco autores, A Method for Hot Spot Temperature Prediction of a $10 \mathrm{kV}$ Oil-Immersed Transformer, https://doi.org/10.1109/ACCESS.2019.2924709, IEEE Access, 7, 107380-107388 (2019).

Estupiñan, J.J., Giral D. y Martínez F., Implementación de algoritmos basados en máquinas de soporte vectorial (SVM) para sistemas eléctricos: revisión de tema, http://dx.doi.org/10.14483/udistrital.jour.tecnura.2016.2.a11, Revista Tecnura, 20(48), 149-170 (2016).

Gómez, V.A., Hernández C. y Rivas E., Visión General, Características y Funcionalidades de la Red Eléctrica Inteligente (Smart Grid), http://dx.doi.org/10.4067/S0718-07642018000200089, Información Tecnológica, 29(2), 89-102 (2018).

Hastie, T., Tibshirani R. y Friedman J., The Elements of Statistical Learning Data Mining, Inference, and Prediction, $2 \mathrm{a}$ Ed., Vol. 2, 1-757, Springer, Stanford, USA (2009). 
IEC 60076-7: International Electrotechnical Commission, Power transformers - Part 7: Loading Guide for Mineral-OilImmersed Power Transformers, 1-89, Ginebra, Suiza (2018).

IEEE Std. C57.91: IEEE Standards Association, Guide for Loading Mineral-Oil-Immersed Transformers and Step-Voltage Regulators, 1-106, New York, USA (2012).

Martínez, D., Céspedes A. y García D., Evaluación de Modelos Térmicos usados para Estimar la Temperatura del Punto Caliente en Transformadores de Distribución, http://dx.doi.org/10.4067/S0718-07642019000300295, Información Tecnológica, 30(3), 295-306 (2019).

Medina, R.D., Romero A.A., Mombello E.E. y Rattá G., Assessing Degradation of Power Transformer Solid Insulation considering Thermal Stress and Moisture Variation, https://doi.org/10.1016/j.epsr.2017.04.006, Electric Power Systems Research, 151, 1-11 (2017).

Medina, R.D., Romero A.A., Mombello E.E. y Rattá G., Comparative Study of Two Thermal Aging Estimating Methods for Power Transformers, https://doi.org/10.1109/TLA.2015.7387233, IEEE Latin America Transactions, 13(10), 32873293 (2015).

Mharakurma, E., Nyakoe G. y Akumu A., Power Transformer Hot Spot Temperatures Estimation based on Multiattributes, ISSN 0973-4562, International Journal of Applied Engineering Research, 14(7), 1584-1592 (2019).

Mosquera, R., Castrillón O.D. y Parra L., Máquinas de Soporte Vectorial, Clasificador Naïve Bayes y Algoritmos Genéticos para la Predicción de Riesgos Psicosociales en Docentes de Colegios Públicos Colombianos, http://dx.doi.org/10.4067/S0718-07642018000600153, Información Tecnológica, 29(6), 153-162 (2018).

Naderian, A., Cress S. y otros tres autores, An Approach to Determine the Health Index of Power Transformers, https://doi.org/10.1109/ELINSL.2008.4570308, IEEE Electrical Insulation, Conference Record of the 2008 IEEE International Symposium, 192-196 (2008).

Qian, T., Tang W. y otros cuatro autores, Comparisons of Transformer Top Oil Temperature Calculation Models using Support Vector Regression Optimised by Genetic Algorithm, https://doi.org/10.1049/oap-cired.2017.0539, IET, 2017(1), 69-73 (2017).

Romero, A.A., Mombello E. y Rattá G., Assessing the Loss-of-Insulation Life of Power Transformers by Estimating their Historical Loads and Ambient Temperature Profiles using ANNs and Monte Carlo Simulations, https://doi.org/10.15446/dyna.v83n197.48134, Dyna, 83(197), 104-113 (2016).

Susa, D. y Lehtonen M., Dynamic Thermal Modeling of Power Transformers: Further Development-Part I, https://doi.org/10.1109/TPWRD.2005.864069, IEEE Transactions on Power Delivery, 21(4), 1961-1970 (2006).

Susa, D. y Lehtonen M., Dynamic Thermal Modeling of Power Transformers: Further Development-Part II, https://doi.org/10.1109/TPWRD.2005.864068, IEEE Transactions on Power Delivery, 21(4), 1971-1980 (2006).

Susa, D., Dynamic Thermal Modelling of Power, Helsinki University of Technology, Dissertations 9, 1-131 (2005).

Susa, D., Lehtonen M. y Nordman H., Dynamic Thermal Modelling of Power Transformers, https://doi.org/10.1109/TPWRD.2004.835255, IEEE Transactions on Power Delivery, 20(1), 197-204 (2005). 